T. Ohsawa

Nagoya Math. J.

Vol. 195 (2009), 41-56

\title{
A REDUCTION THEOREM FOR STABLE SETS OF HOLOMORPHIC FOLIATIONS ON COMPLEX TORI
}

\author{
TAKEO OHSAWA
}

\begin{abstract}
Some complex $n$-tori admit holomorphic foliations of codimension one besides the flat ones. It will be shown that such nonlinear foliations, possibly with singularities, can be reduced to those on 2-tori under some topological conditions. A crucial step is an application of the Hodge theory on pseudoconvex manifolds.
\end{abstract}

\section{Introduction}

Let $M$ be a compact Kähler manifold and let $X \subset M$ be a real-analytic Levi flat hypersurface. It was provided in [O-3] that $M \backslash X$ does not admit a $\mathcal{C}^{\infty}$ plurisubharmonic exhaustion function whose Levi form has everywhere at least 3 positive eigenvalues outside a compact subset of $M \backslash X$. It turned out later that, as well as $M \backslash X$, complements of effective divisors of $M$ with topologically trivial normal bundles have the same non-convexity property as above (cf. [O-4]).

Applying these results for $M=\mathbb{C P}^{n}$, one obtains an alternate proof of a theorem of LinsNeto [LN], asserting in particular that there exist no real analytic Levi flat hypersurfaces in $\mathbb{C P}^{n}$ if $n \geq 3$, because the complements of such hypersurfaces would admit strictly plurisubharmonic exhaustion functions by the positivity of the holomorphic bisectional curvature of the Fubini-Study metric. In view of this, we would like to pursue the consequences of [O-3], [O-4] in case $M$ is a complex torus equipped with a flat metric, by exploiting a formula, due to Matsumoto [M], for the Levi form of distance function to complex submanifolds in $\mathbb{C}^{n}$.

TheOREM 0.1. Let $T$ be a complex torus equipped with a flat Hermitian metric and let $A \subset T$ be a closed subset. Assume that there exist a neighbourhood $U \supset A$ and a one-codimensional holomorphic foliation $\mathcal{F} \subset \Omega_{T}^{1}$

Received July 10, 2007.

Revised August 27, 2007, October 31, 2007, August 12, 2008.

Accepted September 9, 2008.

2000 Mathematics Subject Classification: Primary 32E40; Secondary 53C40. 
(= the sheaf of holomorphic 1-forms on $T$ ) with possibly singular leaves on $U$ such that $A$ is a stable set of $\mathcal{F}$ (i.e. $A$ is the union of leaves of $\mathcal{F}$ intersecting with $A$ ) and that $\mathcal{F}$ is topologically trivial on a neighborhood of $A$. Then, either $\mathcal{F}$ is generated by a holomorphic 1-form on $T$ and $A$ is totally geodesic, or there exist a complex 2-torus $T^{\prime}$, a holomorphic map $\pi: T \rightarrow T^{\prime}$ and a closed subset $A^{\prime} \subset T^{\prime}$ such that $A=\pi^{-1}\left(A^{\prime}\right)$. In the latter case, $\mathcal{F}$ is the pull back of some foliation $\mathcal{F}^{\prime}$ on a neighbourhood of $A^{\prime}$ whenever $A$ is not complex analytic.

The author does not know whether or not $\mathcal{F}^{\prime} \mid A^{\prime}$ is topologically trivial if so is $\mathcal{F} \mid A$ in the above situation besides the trivial case where $A$ is a real hypersurface. Theorem 0.1 shows in particular that the classification of real analytic Levi flat hypersurfaces in complex tori is reduced to that in the 2-tori (See also the remark after the proof of Theorem 0.1).

\section{$\S 1$. Preliminaries}

Let $M$ be a complex manifold. We shall denote by $\Omega_{M}^{p}$ the sheaf of holomorphic $p$-forms on M. By a 1-codimensional holomorphic foliation on $M$ with possibly singular leaves, we shall mean an invertible subsheaf $\mathcal{F}$ of $\Omega_{M}^{1}$ which is locally generated by $\mathrm{d} f$ for some nonconstant holomorphic function $f$. A nonempty connected subset $F \subset M$ will be called a leaf of $\mathcal{F}$ if, for any $p \in M$, there exists a neighbourhood $U \ni p$ and a holomorphic function $f$ on $U$ such that $\mathrm{d} f$ generates $\mathcal{F} \mid U$ and $F \cap U=f^{-1}(B)$ for some subset $B$ of $\mathbb{C}$ with at most countably many elements.

THEOREM 1.1. Let $M$ be a compact Kähler manifold and let $A \subset M$ be a proper and nonempty closed subset. Assume that there exist a neighbourhood $U \supset A$ and a 1-codimensional holomorphic foliation with possibly singular leaves on $U$ say $\mathcal{F}$, such that $A$ is a stable set of $\mathcal{F}$ and that $\mathcal{F}$ is topologically trivial as an invertible sheaf. Then $M \backslash A$ does not admit a $\mathcal{C}^{\infty}$ plurisubharmonic exhaustion function whose Levi form has everywhere at least 3 positive eigenvalues outside a compact subset of $M \backslash A$.

Proof. Suppose that such a function, say $\Phi$, exist. We shall show that $\Phi$ violates the maximum principle.

Since $\mathcal{F}$ is topologically trivial, there exists an element of $H^{1}(U, \mathcal{O})$, say $\alpha$, which is mapped to $\mathcal{F}$ by the exponential homomorphism

$$
H^{1}(U, \mathcal{O}) \longrightarrow H^{1}\left(U, \mathcal{O}^{*}\right)
$$


Then, by Grauert-Riemenschneider's vanishing theorem applied on $M \backslash A$, $\alpha$ can be extended as a cohomology class of $H^{1}(M, \mathcal{O})$.

Therefore, $\mathcal{F}$ can be extended as a topologically trivial invertible sheaf over $M$, say $\tilde{\mathcal{F}}$.

Let us fix a system of open sets $U_{i} \subset U(i=1,2, \ldots, m)$ and holomorphic functions $f_{i}$ on $U_{i}$ in such a way that $\mathrm{d} f_{i}$ generates $\mathcal{F} \mid U_{i}$ and that $A \subset \bigcup_{i=1}^{m} U_{i}$. Then there exists a system of nowhere vanishing holomorphic functions $\left\{e_{i j}\right\}$ on $U_{i} \cap U_{j}$ such that $\mathrm{d} f_{i}=e_{i j} \mathrm{~d} f_{j}$ holds on $U_{i} \cap U_{j}$. This means that the system $\left\{\mathrm{d} f_{i}\right\}$ is identified with an $\mathcal{F}$-valued 1 -form say $\xi$ on a neighbourhood of $A$.

Note that $\xi$ can be extended as an $\mathcal{F}$-valued holomorphic 1-form on $M$ say $\tilde{\xi}$, since a Lefschetz type isomorphism theorem holds on $M \backslash A$ (cf. [O-1], [D], [O-5]).

Since $\mathrm{d} \tilde{\xi}=0$ holds with respect to the unitary flat connection of $\tilde{\mathcal{F}}$, $\tilde{\xi}$ is locally d-exact by Poincaré's lemma. This means that we could have chosen $f_{i}$ in advance so that $U_{i} \cap U_{j}$ are connected and $e_{i j}$ are constants of modulus one. In this situation we have

$$
f_{i}=e^{\sqrt{-1} \theta_{i j}} f_{j}+c_{i j} \quad \text { on } U_{i} \cap U_{j}
$$

for some $\theta_{i j} \in \mathbb{R}$ and $c_{i j} \in \mathbb{C}$.

We put $\Delta=\{(p, p) \mid p \in A\}$ and define a function $\delta$ on a neighbourhood of $\Delta$ in $U \times U$ by letting $\delta(z, w)=\left|f_{i}(z)-f_{i}(w)\right|$ if $(z, w) \in U_{i} \times U_{i}$ for some $i$. Note that $\delta$ is well defined by (1.1).

Then we put

$$
\delta_{A}(z)=\inf \{\delta(z, w) \mid(z, w) \in V \cap(U \times A)\} .
$$

Since $A$ is compact and is the union of leaves of $\mathcal{F}$ intersecting with $A, \delta_{A}$ is continuous on a sufficiently small neighbourhood $W$ of $A$, is constant on every leaf of $\mathcal{F} \mid W$, and satisfies $\delta_{A}^{-1}(0)=A$.

Let us choose $\varepsilon>0$ such that $\delta_{A}^{-1}(\varepsilon)$ has a compact component say $L$ in $W$.

Let $p \in L$ be any point where $\Phi \mid L$ takes its maximum, and let $F_{p}$ be the leaf of $\mathcal{F} \mid W$ passing through $p$. Then $\Phi \mid F_{p}$ must be constant by the maximum principle, but the Levi form of $\Phi \mid F_{p}$ is not zero by the assumption on the Levi form of $\Phi$, provided that $\varepsilon$ is chosen to be sufficiently small. This is the desired contradiction. 


\section{§2. Matsumoto's formula and its consequences}

Let $\mathbb{D}=\{z \in \mathbb{C}|| z \mid<1\}$, let $\gamma: \mathbb{D}^{r} \rightarrow \mathbb{C}^{q}$ be a holomorphic map satisfying $\gamma(0)=0$ and $\mathrm{d} \gamma(0)=0$, and let

$$
G=\left\{(z, \gamma(z)) \mid z=\left(z_{1}, \ldots, z_{r}\right) \in \mathbb{D}^{r}\right\} .
$$

For any point $(z, \zeta) \in \mathbb{D}^{r} \times \mathbb{C}^{q}$, let $\delta(z, \zeta)$ be the euclidean distance from $(z, \zeta)$ to $G$.

We put

$$
\begin{aligned}
& \varphi(z, \zeta)=-\log \delta(z, \zeta) \\
& \Phi(\zeta)=\left.\left(\frac{\partial^{2} \varphi(z, \zeta)}{\partial z_{i} \partial \bar{z}_{j}}\right)_{1 \leq i, j \leq r}\right|_{z=0} \\
& F(z)=\left(\frac{\partial^{2} \gamma}{\partial z_{i} \partial z_{j}}\right)_{1 \leq i, j \leq r}=\left(F_{1}(z), \ldots, F_{q}(z)\right)
\end{aligned}
$$

and

$$
\Lambda(\zeta)=\sum_{\mu=1}^{q} \overline{F_{\mu}(0)} \zeta_{\mu} .
$$

Matsumoto's Formula. (cf. $[\mathrm{M}]$ ) There exists $\varepsilon>0$ such that

$$
\Phi(\zeta)=\frac{1}{2}\|\zeta\|^{-2} \overline{\Lambda(\zeta)} \Lambda(\zeta)\left[E_{r}-\overline{\Lambda(\zeta)} \Lambda(\zeta)\right]^{-1}
$$

holds for $0<\|\zeta\|<\varepsilon$. Here $\|\zeta\|^{2}=\sum_{\mu=1}^{q}\left|\zeta_{\mu}\right|^{2}$ and $E_{r}$ denotes the unit matrix of rank $r$.

If $q=1$, then (2.1) becomes simpler:

$$
\Phi(\zeta)=\frac{1}{2} \overline{F_{1}(0)} F_{1}(0)\left[E_{r}-\overline{\Lambda(\zeta)} \Lambda(\zeta)\right]^{-1} .
$$

We shall put (2.2) in more geometric form below.

Let $D$ be a domain in $\mathbb{C}^{n}$ and let $Y$ be a closed complex submanifold of codimension one in $D$.

For each $p=\left(p_{1}, \ldots, p_{n}\right) \in Y$, we take unitary matrix $A=\left(A_{i j}\right)$ of rank $n$ in such a way that the vectors

$$
\sum_{j=1}^{n} \overline{A_{i j}} \frac{\partial}{\partial z_{j}} \quad(2 \leq i \leq n)
$$


belong to the holomorphic tangent space of $Y$ at $p$.

By letting

$$
\begin{aligned}
\zeta & =\sum_{j=1}^{n} A_{1 j}\left(z_{j}-p_{j}\right) \\
u_{i} & =\sum_{j=1}^{n} A_{i+1}\left(z_{j}-p_{j}\right) \quad(1 \leq i \leq n-1)
\end{aligned}
$$

and $u=\left(u_{1}, \ldots, u_{n-1}\right)$, we define a holomorphic function $h$ on a neighbourhood of $u=0$ in such a way that $Y$ is defined by $\zeta=h(u)$ on a neighbourhood of $p$.

Since the hypersurface $\zeta=0$ is tangent to $Y$ at $(\zeta, u)=(0,0)$, we have $h(0)=0$ and $\mathrm{d} h(0)=0$.

Then we put

$$
H_{p}=\left.\left(\frac{\partial^{2} h}{\partial u_{i} \partial u_{j}}\right)_{1 \leq i, j \leq n-1}\right|_{u=0}
$$

and

$$
Q_{p}=\left(Q_{i j}\right)_{1 \leq i, j \leq n-1}=H_{p} \overline{H_{p}} .
$$

Let $\mathcal{M}_{Y}$ denote a semipositive $(1,1)$-form on $Y$ defined by the correspondence

$$
p \longmapsto \sqrt{-1} \sum_{i, j=1}^{n-1} Q_{i j} \mathrm{~d} u_{i} \wedge \mathrm{d} \bar{u}_{j} .
$$

Let $U$ be a tubular neighbourhood of $Y$ with an orthogonal retraction $\rho: U \rightarrow Y$, and let $\mathcal{M}_{U}$ be the $(1,1)$-form on $U$ which is induced from $\mathcal{M}_{Y}$ by the parallel transport along the line segments which are perpendicular to $Y$.

Then, from (2.2) we deduce the following.

Proposition 2.1. Let $Y, U$ and $\mathcal{M}_{U}$ be as above, and let $\delta_{Y}(z)$ be the distance from $z$ to $Y$. Then there exists a neighbourhood $V \supset Y$ such that $V \subset U$ and

$$
2 \sqrt{-1} \partial \bar{\partial}\left(-\log \delta_{Y}\right) \geq \mathcal{M}_{U}
$$

holds on $V \backslash Y$. 
By an abuse of language, real (1,1)-forms $\sqrt{-1} \sum_{i, j=1}^{n} \Theta_{i j} \mathrm{~d} z_{i} \wedge \mathrm{d} \bar{z}_{j}$ $\left(\Theta_{i \bar{j}}=\overline{\Theta_{j \bar{i}}}\right)$ will be identified with a Hermitian form $\sum_{i, j} \Theta_{i \bar{j}} \xi^{i} \overline{\xi^{j}}$ on the space of holomorphic tangent vectors $\sum_{i} \xi^{i} \frac{\partial}{\partial z_{i}}$ of $\mathbb{C}^{n}$. Similar identification will be carried over on complex manifolds, too.

In order to exploit (2.2) in full strength, we observe that (2.3) holds with respect to any flat Hermitian metric on $\mathbb{C}^{n}$ and that the sums of functions $-\log \delta_{Y}$ for several metrics have more convexity than the single one has.

To put it into a proposition, let $T_{z}^{1,0} \mathbb{C}^{n}$ denote the holomorphic tangent space of $\mathbb{C}^{n}$ at $z$. For any two points $z, z^{\prime} \in \mathbb{C}^{n}$, let

$$
\tau_{z^{\prime}}^{z}: T_{z}^{1,0} \mathbb{C}^{n} \longrightarrow T_{z^{\prime}}^{1,0} \mathbb{C}^{n}
$$

be the parallel transport.

For any $z \in U$, let $N_{z}$ be the maximal complex linear subspace of $T_{z}^{0,1} \mathbb{C}^{n}$ satisfying $\mathcal{M}_{U} \mid N_{z}=0$, and put

$$
\mathcal{N}_{z}=\lim _{W} \bigcap_{z^{\prime} \in W} \tau_{z}^{z^{\prime}}\left(N_{z^{\prime}}\right)
$$

Here $W$ runs through the neighbourhoods of $z$.

By the analyticity of $Y$, it is clear that $\mathcal{N}_{z}$ are parallel to each other on any connected component of $U$ and that $\operatorname{dim} \mathcal{N}_{z}$ is locally constant on $U$.

Similarly we define, given a Hermitian metric of the form

$$
g=\sum_{i, j=1}^{n} g_{i j} \mathrm{~d} z_{i} \mathrm{~d} \bar{z}_{j} \quad\left(g_{i j} \in \mathbb{C}\right)
$$

and a tubular neighbourhood $U^{\prime}$ of $Y$ with an orthogonal projection $U^{\prime} \rightarrow Y$ with respect to $g$, the associated $(1,1)$-form $\mathcal{M}_{U^{\prime}}=\mathcal{M}_{U^{\prime}, g}$, its null space $N_{z}=N_{z, g}$ and the space $\mathcal{N}_{z}=\mathcal{N}_{z, g}$ as above for $z \in U^{\prime}$.

Clearly $N_{z, g}$ may depend on $g$ if $z \notin Y$ because so does the field of normals for $Y$. However, as is clear from the definition, $\mathcal{N}_{z}$ does not depend on the choice of $g$.

For any subset $B \subset \mathbb{C}^{n}$, let $\delta_{B, g}$ denote the distance to $B$ with respect to $g$. Note that, for any $z \in U \backslash Y$ and $z^{\prime} \in Y$ such that $\delta_{Y, g}(z)$ is attained by the distance from $z$ to $z^{\prime}$, the foot of perpendicular from $z$ to $Y$ runs through a dense subset of a neighbourhood of $z^{\prime}$ in $Y$ as $g$ varies in the set of metrics of the form (2.4).

Hence we deduce the following from Proposition 2.1. 
Proposition 2.2. Let $Y \subset D \subset \mathbb{C}^{n}$ be as above and let $x \in Y$. Then there exists a neighbourhood $U_{0} \ni x$ in $\mathbb{C}^{n}$, a positive number $c$, and flat Hermitian metrics $g_{1}, \ldots, g_{m}$ of the form (2.4), such that the function

$$
\psi=\sum_{k=1}^{m}\left(\log \delta_{Y, g_{k}}\right)^{2}
$$

satisfies the following.

$$
\begin{aligned}
& \text { The eigenvalues } \gamma_{1} \leq \gamma_{2} \leq \cdots \leq \gamma_{n} \text { of } \sqrt{-1} \partial \bar{\partial} \psi \text { at } z \text { satisfy } \\
& \gamma_{k}>c \text { for } k>\operatorname{dim} \mathcal{N}_{z} \text { if } z \in U_{0} \backslash Y .
\end{aligned}
$$

It is clear that Proposition 2.2 and the preceding materials carry over if $Y$ is a locally closed complex submanifold of codimension one in a complex torus equipped with flat Hermitian metrics.

Moreover, if $Y$ depends continuously on a parameter $t \in \mathbb{D}$ and forms a family of complex submanifolds of codimension one say

$$
\mathcal{Y}=\left\{Y_{t}\right\}_{t \in \mathbb{D}} \quad \text { with } \quad Y_{0}=Y
$$

in some fixed domain, then $U_{0}, c$ and $\psi=\psi_{t}$ in Proposition 2.2 can be chosen in such a way that (2.5) holds on $U_{0} \backslash Y_{t}$ for $\psi_{t}$ for all sufficiently small $t$.

From now on, let $T$ be a complex $n$-torus and let $A \subset T$ be a nonempty and closed subset. We shall assume that there exists a neighbourhood $U \supset A$ and a 1-codimensional holomorphic foliation $\mathcal{F}$ on $U$ with possibly singular leaves such that $A$ is stable, i.e. $A$ is the union of leaves of $\mathcal{F}$ intersecting with $A$.

For each point $a \in A$, let $U_{a}$ be a neighbourhood of $a$ in $T$ such that $\mathcal{F} \mid U_{a}$ is generated by $\mathrm{d} f_{a}$ for some holomorphic function $f_{a}$ on $U_{a}$.

We put

$$
A_{0}=\left\{a \in A \mid \mathrm{d} f_{a}=0 \text { at } a\right\}
$$

and

$$
A^{\circ}=A \backslash A_{0} .
$$

Clearly $A_{0}$ is nowhere dense in $A$.

Let $\mathrm{Herm}^{+}(T)$ denote the set of flat Hermitian metrics on $T$. By fixing a basis of the space of global holomorphic vector fields on $T$, we may identify $\mathrm{Herm}^{+}(T)$ with a subset of the space of Hermitian matrices. 
For any $g \in \operatorname{Herm}^{+}(T)$ and $z \in T \backslash A$ we put

$$
A_{z, g}=\left\{w \in A \mid \operatorname{dist}_{g}(z, A)=\operatorname{dist}_{g}(z, w)\right\},
$$

where $\operatorname{dist}_{g}($,$) denotes the distance with respect to g$. We put $\delta_{g}(z)=$ $\operatorname{dist}_{g}(z, A)$ for simplicity.

If $w \in A_{z, g}$, then there exists a flat holomorphic curve $l_{w}: \mathbb{C} \rightarrow T$ such that $l_{w}(0)=z, l_{w}(1)=w$ and $l_{w}^{1}(A) \cap \mathbb{D}=\emptyset$. We shall say that $z$ is generic with respect to $(A, g)$ if $A_{z, g} \subset A^{\circ}$ and $l_{w}$ intersects with the leaf passing through $w$ transversally at $w$ for any $w \in A_{z, g}$. Then we put

$$
A_{g}^{*}=\{z \in T \backslash A \mid z \text { is generic with respect to }(A, g)\} .
$$

Clearly $A_{g}^{*}$ is nonempty.

Proposition 2.3. There exists a neighbourhood $W \supset A$ such that, for any point $z \in W \backslash A$, there exists a $g \in \operatorname{Herm}^{+}(T)$ such that $z \in A_{g}^{*}$.

Proof. Let $a \in A$ be any point, let $\left(U_{a}, f=f_{a}\right)$ be as above, and let $Y=f^{-1}(f(a))$. If $a$ is a singular point of $Y$, then there exist neighbourhoods $W^{(k)}(k=1,2, \ldots, m)$ of $a$ and surjective holomorphic maps

$$
\pi_{k}: W^{(k)} \longrightarrow \mathbb{D}^{n-1}
$$

satisfying the following.

1. $\pi_{k}^{-1}\left(z^{\prime}\right)$ are flat submanifolds of $T$ for all $z^{\prime} \in \mathbb{D}^{n-1}$.

2. $W^{(k)} \cap f^{-1}\left(f\left(a^{\prime}\right)\right)(k=1,2, \ldots, m)$ are nonsingular if $f\left(a^{\prime}\right) \neq f(a)$.

3. $\pi \mid W^{(k)} \cap Y$ is a finite morphism for every $k$.

4. $\bigcap_{k=1}^{m} W^{(k)} \cap \pi_{k}^{-1}\left(\pi_{k}\left(Y_{k}\right)\right) \subset A$, where $Y_{k}$ denotes the set $\left\{z \in W^{(k)} \cap Y\right.$ $\pi_{k}^{-1}\left(\pi_{k}(z)\right)$ does not intersect transversally with $Y$ at $\left.z\right\}$.

Then it is easy to see that there exists a neighbourhood $W_{a} \ni a$ such that $W_{a} \subset \bigcap_{k=1}^{m} W^{(k)}$ and that, for any point $z \in W_{a} \backslash A$, there exists a $g \in \operatorname{Herm}^{+}(T)$ such that $z \in A_{g}^{*}$.

Let $g_{1}, \ldots, g_{m} \in \operatorname{Herm}^{+}(T)$. For any $a \in A$, let $\left(U_{a}, f\right)$ be chosen as above and put

$$
\psi_{g_{1}, \ldots, g_{m}, a}(z)=\sup \left\{\sum_{k=1}^{m}\left(\log \delta_{f^{-1}(\zeta), g_{k}}(z)\right)^{2} \mid \zeta \in f\left(U_{a}\right)\right\} .
$$


Let $U^{\prime}{ }_{a}$ be any neighbourhood of $a$ satisfying $U^{\prime}{ }_{a} \Subset U_{a}$. Since $A$ is compact, there exist finitely many points $a_{1}, \ldots, a_{\mu} \in A$ such that $A \subset \bigcup_{i=1}^{\mu} U^{\prime} a_{i}$.

Then it is easy to see that there exists a neighbourhood $V \supset A$ such that

$$
\psi_{g_{1}, \ldots, g_{m}, a_{i}}(z)=\psi_{g_{1}, \ldots, g_{m}, a_{j}}(z)
$$

holds for any $z \in V \cap U^{\prime}{ }_{a_{i}} \cap U^{\prime}{ }_{a_{j}} \backslash A$.

Hence we obtain a well defined function $\psi_{g_{1}, \ldots, g_{m}}$ on $V \backslash A$ by setting $\psi_{g_{1}, \ldots, g_{m}}(z)=\psi_{g_{1}, \ldots, g_{m}, a_{i}}(z)$ on $V \cap U^{\prime}{ }_{a_{i}} \backslash A$.

In order to describe a property of the function $\psi_{g_{1}, \ldots, g_{m}}$, we shall introduce a quantity $\nu_{A}$ below.

For any $z \in A_{g}^{*}$ and $w \in A_{z, g}$, let $I_{z, w}$ be the geodesic interval joining $z$ and $w$. We fix a neighbourhood $U_{z, w} \supset I_{z, w}$ in such a way that there exists an orthogonal retraction from $U_{z, w}$ to the leaf of $\mathcal{F} \mid U_{z, w} \cap U$ containing $w$, say $F_{z, w}$.

Then, with respect to the pair $\left(F_{z, w}, U_{z, w}\right)$, we define the subspaces $N_{z^{\prime}}$ and $\mathcal{N}_{z^{\prime}}$ of $T_{z^{\prime}}^{1,0} T$ for any $z^{\prime} \in U_{z, w}$ similarly as in the case of the pairs $\left(Y, U^{\prime}\right)$.

Denoting $\mathcal{N}_{z^{\prime}}$ by $\mathcal{N}_{z^{\prime}, w}$ to make the dependence of $\mathcal{N}_{z^{\prime}}$ on $w$ explicit, we put

$$
\mathcal{N}_{A, z}=\lim _{\mathscr{U}} \bigcap_{g^{\prime} \in \mathcal{U}} \bigcap_{w \in A_{z, g^{\prime}}} \mathcal{N}_{z, w}
$$

and

$$
\nu_{A}=\liminf _{U^{\prime}} \inf \left\{\operatorname{dim} \mathcal{N}_{A, z} \mid z \in U^{\prime} \backslash A\right\} .
$$

Here $\mathcal{U}$ runs through the neighbourhoods of $g$ and $U^{\prime}$ runs through the neighbourhoods of $A$. Clearly $\mathcal{N}_{A, z^{\prime}}$ and $\mathcal{N}_{A}$ do not depend on the choice of $g$ if $A$ contains a leaf of $\mathcal{F}$ densely.

Now, with respect to the function $\psi_{g_{1}, \ldots, g_{m}}$ as above, it is easy to see that the following is true.

Proposition 2.4. If some leaf of $\mathcal{F}$ is dense in $A$, then there exist $g_{1}, \ldots, g_{m} \in \operatorname{Herm}^{+}(T)$, a neighbourhood $V \supset A$, a point $z \in V \backslash A, a$ neighbourhood $\Omega$ of $z$, and a $\mathcal{C}^{\infty}$ semipositive $(1,1)$-form $\theta$ on $\Omega$ such that $\theta^{n-\nu_{A}}$ has no zeros on $\Omega$ and that $\sqrt{-1} \partial \bar{\partial} \psi_{g_{1}, \ldots, g_{m}}-\theta$ is a semipositive $(1,1)$-current on $\Omega$.

In order to proceed further, we need the following approximation theorem. 
THEOREM 2.1. Let $X$ be a complex manifold admitting a (not necessarily complete) flat Hermitian metric, let $\varphi$ be a (not necessarily continuous) plurisubharmonic function on $X$ such that the sublevel sets $X_{c}:=\{x \in X \mid$ $\varphi(x)<c\}$ are relatively compact for all $c \in \mathbb{R}$, and let $r \in \mathbb{N}$. Suppose that there exist a nonempty open set $\Omega \subset X$ and a $\mathcal{C}^{\infty}$ semipositive $(1,1)$-form $\theta$ on $\Omega$ such that $\theta^{r} \neq 0$ and that $\sqrt{-1} \partial \bar{\partial} \varphi-\theta$ is a semipositive current on $\Omega$. Then there exists a $\mathcal{C}^{\infty}$ plurisubharmonic exhaustion function $\tilde{\varphi}$ on $X$ whose Levi form has everywhere at least $r$ positive eigenvalues.

Proof. Let us fix $c_{0} \in \mathbb{R}$ such that $X_{c_{0}} \cap\left\{\theta^{r} \neq 0\right\} \neq \emptyset$. Since $X_{c}$ are all relatively compact in $X$, there exist $c_{i}(i=1,2,3,4)$ such that $\overline{X_{c_{i-1}}} \subset X_{c_{i}}(i=1,2,3,4)$. Let $\mathcal{X}$ be a non-negative $\mathcal{C}^{\infty}$ function on $\mathbb{R}$ such that supp $\mathcal{X} \subset[-1,1]$ and $\int_{-\infty}^{\infty} \mathcal{X}(t) \mathrm{d} t=1$. Let $d(x, y)$ be the distance between $x$ and $y$ in $X$. For any $\varepsilon>0$ we put $\rho_{X, \varepsilon}(y)=\varepsilon^{-2 n}(d(x, y) / \varepsilon)$.

Then there exist $\varepsilon_{0}>0$ and a constant $C$ such that supp $\rho_{X, \varepsilon} \Subset X$ and $\int_{X} \rho_{X, \varepsilon}(y) \omega^{n}=C$ hold for $0<\varepsilon<\varepsilon_{0}$ and $x \in \overline{X_{c_{4}}}$. Here $\omega\left(=\omega_{y}\right)$ denotes the fundamental form of the flat metric on $X$.

Given any locally integrable function $\psi$ on $X$ we put

$$
\psi_{\varepsilon}(x)=\frac{1}{C} \int_{X} \psi(y) \rho_{X, \varepsilon}(y) \omega^{n} .
$$

Then $\varphi_{\varepsilon}$ is plurisubharmonic on $X_{c_{4}}$ and one may choose $\varepsilon_{0}$ in advance so that $c_{0}<\varphi_{\varepsilon}$ holds on $\overline{X_{C_{2}}} \backslash X_{C_{1}}$ and $\varphi_{\varepsilon}<c_{4}$ holds on $X_{c_{3}}$.

Let $\lambda$ be a $\mathcal{C}^{\infty}$ convex increasing function on $\mathbb{R}$ such that $\lambda(t)=c_{0}$ on $\left(-\infty, c_{2}\right)$ and $\lambda\left(\left(c_{2}+c_{3}\right) / 2\right)>c_{4}$. Then we put

$$
\varphi_{\varepsilon}^{\vee}(x)= \begin{cases}\max \left\{\varphi_{\varepsilon}(x), \lambda(\varphi(x))\right\} & \text { if } x \in X_{c_{3}} \\ \lambda(\varphi(x)) & \text { if } x \in X \backslash X_{c_{3}} .\end{cases}
$$

Clearly $\varphi_{\varepsilon}^{\vee}$ is a plurisubharmonic function on $X$. Note that $\varphi_{\varepsilon}^{\vee}$ is $\mathcal{C}^{\infty}$ on $X_{c_{1}}$ and the set

$$
\Omega\left(\varphi_{\varepsilon}^{\vee}\right):=\left\{x \in X_{c_{1}} \mid\left(\partial \bar{\partial} \varphi_{\varepsilon}^{\vee}\right)^{r}(x) \neq 0\right\}
$$

contains $\Omega^{\varepsilon}:=\left\{x \in X_{c_{1}} \mid d(x, U)<\varepsilon\right\}$ for any $0<\varepsilon<\varepsilon_{0}$. Here $d(x, \Omega)$ denotes the distance from $x$ to $\Omega$.

We put $\varphi^{\delta}=\varphi+\delta \varphi_{\varepsilon}^{\vee}$ for $\delta>0$. Then $\varphi^{\delta}$ are plurisubharmonic on $X$ and satisfy $c_{0}<\left(\varphi^{\delta}\right)_{\varepsilon}$ on $\overline{X_{c_{0}}} \backslash X_{c_{1}}$ and $\left(\varphi^{\delta}\right)_{\varepsilon}<c_{4}$ on $X_{c_{3}}$ for sufficiently small $\delta$. 
Therefore, by taking the maximum of $\left(\varphi^{\delta}\right)_{\varepsilon}$ and $\lambda(\varphi(x))$ as in (2.6), we obtain a plurisubharmonic function $\left(\varphi^{\delta}\right)_{\varepsilon}^{\vee}$ such that $\Omega\left(\left(\varphi^{\delta}\right)_{\varepsilon}^{\vee}\right) \supset \Omega^{2 \varepsilon}$ holds true.

Hence, by putting further $\varphi^{\delta, \delta^{\prime}}=\varphi^{\delta}+\delta^{\prime}\left(\varphi^{\delta}\right)_{\varepsilon}^{\vee}$ for $\delta^{\prime}>0$ and repeating the above smoothing procedure one after another, we arrive at a plurisubharmonic function $\varphi_{*}=\left(\varphi^{\delta_{1}, \delta_{2}, \ldots, \delta_{m}}\right)_{\varepsilon}^{\vee}$ on $X$ such that $\varphi_{*}$ is $\mathcal{C}^{\infty}$ on $X_{c_{1}}$ and $\Omega\left(\varphi_{*}\right)=X_{c_{1}}$.

The rest is routine and may well be left to the reader.

Thus, combining Matsumoto's formula with Theorem 1.1 and Theorem 2.1, we conclude the following.

Proposition 2.5. Let $T$ be a complex $n$-torus and let $A$ be a nonempty and proper closed subset of $T$. Suppose that there exist a neighbourhood $U \supset A$ and a holomorphic 1-codimensional foliation $\mathcal{F}$ with possibly singular leaves on $U$ whose normal bundle is topologically trivial such that $A$ is stable for $\mathcal{F}$. Then $\nu_{A} \geq n-2$ for any $g \in \operatorname{Herm}^{+}(T)$.

\section{§3. Proof of Theorem 0.1}

If $A=T$, there is nothing to prove. Nevertheless we note that the leaves of $\mathcal{F}$ are all flat in this case because $\mathcal{F}$ is, being topologically trivial on $T$, analytically trivial on $T$ as a subsheaf of $\Omega_{T}^{1}$. If $A=\emptyset$ there is also nothing left to prove.

If $A \neq T, \emptyset$, let $\mathcal{L}_{A}$ be the collection of leaves of $\mathcal{F}$ that are contained in $A$, let $\mathcal{A}=\left\{\bar{\ell} \mid \ell \in \mathcal{L}_{A}\right\}$, and let $A_{*}$ be any element of $\mathcal{A}$. We assume that $A$ is connected, since the general case follows without any difficulty from this case.

Then, in view of the definition of $\mathcal{N}_{A_{*}, z}$ and Proposition 2.5, we know that there exists a trivial subbundle $F \subset T^{1,0} T$ of rank $n-1$ or $n-2$ such that $F_{z}=\mathcal{N}_{A_{*}, z}$ holds for all $z \in T \backslash A_{*}$.

If rank $F=n-1$ for some $A_{*}$, then clearly $A_{*}$ are $(n-1)$-subtori of $T$ and they are parallel to each other. Hence this falls into the second case in the assertion.

If $\operatorname{rank} F=n-2$ for all $A_{*}$, then some $A_{*}$ are not flat. Therefore the foliations defined by $F$ on the leaves of $\mathcal{F}$ in such $A_{*}$ have nowhere dense leaves. In view of the triviality of $F$, this means that $A_{*}$ is the union of parallel translates of an $(n-2)$-subtorus of $T$.

Therefore there exists a complex 2-torus $T^{\prime}$, a surjective holomorphic map $\pi: T \rightarrow T^{\prime}$ and $A_{*}^{\prime} \subset T^{\prime}$ such that $\pi^{-1}\left(A_{*}^{\prime}\right)=A_{*}$. 
If $A_{* *} \in \mathcal{A}$ and $A_{* *} \neq A_{*}$, then we have another 2-torus $T^{\prime \prime}$, a holomorphic map $\pi^{\prime}: T \rightarrow T^{\prime \prime}$ and $A_{* *}^{\prime} \subset T^{\prime \prime}$ such that $\pi^{\prime-1}\left(A_{* *}^{\prime}\right)=A_{* *}$.

However, fibers of $\pi \mid A_{*}$ and $\pi^{\prime} \mid A_{* *}$ must be parallel to each other, since otherwise we would have an exhaustion function on $T \backslash\left(A_{*} \cup A_{* *}\right)$ with a property contradicting Theorem 1.1 (just by adding exhaustion functions of $T \backslash A_{*}$ and $T \backslash A_{* *}$ resulting from Proposition 2.4 and Theorem 2.1).

Consequently, the map $\pi: T \rightarrow T^{\prime}$ pulls back some $A^{\prime} \subset T^{\prime}$ to $A$.

In view of the uniqueness theorem for holomorphic functions, the last assertion of Theorem 0.1 is a corollary of the rest.

Remark. See [O-2] and the appendix following this section for some examples of nonflat foliations on certain tori which admit stable real hypersurfaces. Since smooth real hypersurfaces have topologically trivial analytic normal bundles, holomorphic foliations of codimension one on complex tori, possibly with singular leaves, are reduced to those on 1-tori or 2-tori whenever they have Levi flat stable sets with nonlinear leaves.

\section{§4. Appendix}

The geometric structure of our reduction map $\pi$ is illustrated below by an explicitly described class of Levi flat hypersurfaces living in principal torus bundles over compact Riemann surfaces.

Let $C$ be a compact Riemann surface and let $\pi: \mathcal{T} \rightarrow C$ be a holomorphic principal $n$-torus bundle. Let $T$ be an $n$-torus as the structure group of the bundle and let $\mathfrak{g}$ be the Lie algebra of $T$. The kernel of the exponential map exp: $\mathfrak{g} \rightarrow T$ will be denoted by $\mathfrak{g}_{0}$. We put $\exp \zeta=[\zeta]$ for simplicity. $\mathfrak{g} / \mathfrak{g}_{0}$ and $T$ will not be distinguished below.

Recall that a meromorphic connection on $\mathcal{T} \rightarrow C$ is by definition a system of $\mathfrak{g}$-valued meromorphic 1 -forms, say, $\left\{\omega_{\alpha}\right\}_{\alpha \in A}$ associated to an open covering $\left\{U_{\alpha}\right\}_{\alpha \in A}$ of $C$ with local trivializations

$$
\varphi_{\alpha}: \pi^{-1}\left(U_{\alpha}\right) \longrightarrow U_{\alpha} \times T
$$

$\omega_{\alpha}$ being defined on $U_{\alpha}$, such that $\omega_{\alpha}$ and $\omega_{\beta}$ are related on $U_{\alpha} \cap U_{\beta}$ by $\omega_{\alpha}-\omega_{\beta}=\mathrm{d} c_{\alpha \beta}$. Here $c_{\alpha \beta}$ is defined by

$$
\varphi_{\alpha} \circ \varphi_{\beta}^{-1}(z,[\zeta])=\left(z,\left[\zeta+c_{\alpha \beta}(z)\right]\right)
$$

Meromorphic connections will be simply denoted by $\omega$, by abbreviating $\left\{\omega_{\alpha}\right\}_{\alpha \in A}$. By an abuse of notation, $\omega$ will also stand for $\omega_{\alpha}$. 
Given any closed subgroup $\Gamma \subset T$ and any finite subset $\Sigma \subset C$, we shall denote by $\Omega(\Gamma, \Sigma)$ the set of meromorphic connections $\omega$ of $\mathcal{T} \rightarrow C$ satisfying the following conditions.

1. $\omega$ has at most simple poles contained in $\Sigma$.

2. The holonomy group of $\omega$, as that of a holomorphic connection over $C \backslash \Sigma$, preserves $\Gamma$.

For any $\omega \in \Omega(T, \Sigma)$ and for any $C^{1}$-smooth curve $\gamma:[0,1] \rightarrow C \backslash \Sigma$, let $\omega^{\gamma}$ denote the parallel transport from $\pi^{-1}(\gamma(0))$ to $\pi^{-1}(\gamma(1))$ along $\gamma$ with respect to $\omega$.

If $\gamma$ is a closed curve, $\omega^{\gamma}$ depends only on the homology class $[\gamma]$ of $\gamma$ in $H_{1}(C \backslash \Sigma, Z)$, and is a parallel translate in $\pi^{-1}(\gamma(0))$. We define $P(\omega, \gamma) \in T$ by

$$
\omega^{\gamma}(x)=x+P(\omega, \gamma) \quad\left(x \in \pi^{-1}(\gamma(0))\right)
$$

Then we have

$$
\Omega(\Gamma, \Sigma)=\left\{\omega \mid P(\omega, \gamma) \in T \text { whenever }[\gamma] \in H_{1}(C \backslash \Sigma, Z)\right\}
$$

LEMmA 4.1. $\Omega(\Gamma, \Sigma) \neq \emptyset$ if there exist $\omega \in \Omega(T, \Sigma)$ and a $\mathfrak{g}$-valued meromorphic 1-form $\sigma$ on $C$ with at most simple poles contained in $\Sigma$, such that

$$
\int_{\gamma} \sigma \in P(\omega, \gamma)+\Gamma
$$

holds for any $[\gamma] \in H_{1}(C \backslash \Sigma, Z)$.

Proof. The assertion is obvious because $\omega-\sigma \in \Omega(\Gamma, \Sigma)$.

Proposition 4.1. $\Omega(T, \Sigma) \neq \emptyset$ if $\Sigma \neq \emptyset$.

Proof. It suffices to show that $\Omega(T,\{P\}) \neq \emptyset$ for any $P \in C$. To see this, let $U$ be a coordinate neighbourhood of $P$ and let $\varphi: \pi^{-1}(U) \stackrel{\sim}{\longrightarrow} U \times T$ and $\psi: \pi^{-1}(C \backslash\{P\}) \stackrel{\sim}{\longrightarrow} C \backslash\{P\} \times T$ be respectively local trivializations of $\mathcal{T}$ over $U$ and $C \backslash\{P\}$. Existence of $\varphi$ and $\psi$ is guaranteed by that of holomorphic sections of $\mathcal{T}$ over $U$ and $C \backslash\{P\}$, which is a consequence of the Oka-Grauert principle asserting, in particular, that every holomorphic vector bundle over a noncompact Riemann surface is trivial (cf. [F]). 
Let us define $c: U \backslash\{P\} \rightarrow \mathfrak{g}$ by

$$
\psi \circ \varphi^{-1}(x)=(\pi(x), x+[c(\pi(x))]) .
$$

Since $H^{1,1}(C,[P])=0$, there exist a neighbourhood $V \ni P$ with $\bar{V} \subset U$, a $\mathfrak{g}$-valued holomorphic 1-form $\tau_{0}$ on $C \backslash\{P\}$ and a $\mathfrak{g}$-valued meromorphic 1-form $\tau_{1}$ on $U$ with at most one simple pole at $P$ such that $\mathrm{d} c=\tau_{1}-\tau_{0}$ holds on $U \backslash \bar{V}$.

Hence $\tau=\left\{\tau_{0}, \tau_{1}\right\}$ gives a meromorphic connection on $\mathcal{T} \rightarrow C$ with possibly one simple pole at $P$.

In view of the above construction of the connection $\tau$, it is easy to see that the period of the primitive of $\tau_{1}$ around $P$, denoted by

$$
\oint_{P} \tau \quad\left(=\oint_{P} \tau_{1}\right)
$$

does not depend on the choise of $\varphi$ and $\psi$.

Proposition 4.2. $\oint_{P} \tau$ does not depend on $P$.

Proof. Let $P, Q \in C$ be any distinct points. Then, as is well known, there exists a meromorphic 1-form $\sigma$ on $C$ whose poles are simple and situated at $P$ and $Q$, such that

$$
\oint_{P} \sigma=1 .
$$

Then the meromorphic connection $\tau^{\prime}=\tau-\left(\oint_{P} \tau\right) \sigma$ belongs to $\Omega(T,\{Q\})$ and $\oint_{P} \tau=\oint_{Q} \tau^{\prime}$ holds since $\oint_{Q} \sigma=-1$.

From now on, we shall call the element $\oint_{P} \tau$ of $\mathfrak{g}$ the curvature of the bundle $\mathcal{T} \rightarrow C$ and denote it by $\Theta_{\mathcal{T}}$.

Proposition 4.3. For any closed subgroup $\Gamma \subset T$, there exists $\nu_{0} \in \mathbb{N}$ such that for any integer $\nu \geq \nu_{0}$ one can find a finite set $\Sigma \subset C$ with $\operatorname{deg} \Sigma$ $(:=$ the cardinality of $\Sigma)=\nu$ and $\Omega(\Gamma, \Sigma) \neq \emptyset$.

Proof. Since $\Omega(T, \Sigma) \neq \emptyset$ if $\Sigma \neq \emptyset$ by Proposition 2.1, the conclusion follows from the well known existence theorem for meromorphic differentials of the third kind on $C$ with prescribed periods (cf. $[\mathrm{F}]$ ). 
By the same reasoning as above we obtain the following.

Proposition 4.4. For any closed subgroup $\Gamma \subset T$ satisfying $\left[\sqrt{-1} \mathbb{R} \Theta_{\mathcal{T}}\right] \subset \Gamma$ and $\left[\mathbb{R} \Theta_{\mathcal{T}}\right] \cap \Gamma=\mathbb{Z} \Theta_{\mathcal{T}}$, there exist $\nu_{1} \in \mathbb{N}$ such that one can find, for every integer $\nu \geq \nu_{1}$, a finite set $\Sigma \subset C$ with $\operatorname{deg} \mathbb{Z}=\nu$ and an element $\omega \in \Omega(\Gamma, \Sigma)$ satisfying the following conditions for every $P \in \Sigma$.

1. $\sqrt{-1} \mathbb{R} \oint_{P} \omega \subset \Gamma$

2. $\mathbb{R} \oint_{P} \omega \cap \Gamma=\mathbb{Z} \cdot \frac{1}{2} \oint_{P} \omega$.

(In [O-2], Supplement, Theorem $1, \frac{1}{2}$ is missing in the condition corresponding to 2.)

If $\Gamma$ is a subtorus of real codimension one satisfying the above requirement of Proposition 2.4, then it is easy to see that, for any $x \in \mathcal{T} \backslash \pi^{-1}(\Sigma)$, the closure of the set

$$
\bigcup_{\gamma} \omega^{\gamma}(x+\Gamma) .
$$

where $\gamma$ runs through the $\mathcal{C}^{1}$-smooth curves $\gamma:[0,1] \rightarrow C \backslash \Sigma$ with $\gamma(0)=$ $\pi(x)$, is a real-analytic Levi-flat in $\mathcal{T}$. We shall denote this Levi-flat by $X(\mathcal{T}, \Gamma, \omega, x)$.

We note, as one sees from Proposition 4.4, that $X(\mathcal{T}, \Gamma, \omega, x)$ exists if and only if the curvature $\Theta_{\mathcal{T}}$ of the bundle satisfies

$$
\sqrt{-1} \mathbb{R} \Theta_{\mathcal{T}} \cap \mathfrak{g}_{0} \neq\{0\}
$$

Hence, in particular, flat torus bundles over compact Riemann surfaces always admit Levi-flats of the form $X(\mathcal{T}, \Gamma, \omega, x)$ which are generically fibered over $C$.

Remark. The notation of the curvature as above immediately generalizes to arbitrary principal complex Lie group bundles. However the author does not know how this notion carries over to bundles with higher dimensional base spaces.

Acknowledgements. The author is grateful to the referee for the useful comments which improved the presentation of the paper. 


\section{REFERENCES}

[D] J. P. Demailly, Cohomology of q-convex spaces in top degrees, Math. Z., 204 (1990), 283-295.

[F] O. Foster, Lectures on Riemann surfaces, GTM, Springer, 1980.

[LN] A. LinsNeto, A note on projective Levi flats and minimal sets of algebraic foliations, Ann. Inst. Fourier, 49 (1999), 1369-1385.

[M] K. Matsumoto, Levi form of logarithmic distance to complex submanifolds and its application to developability, Complex Analysis in Several Variables - Memorial Conference of Kiyoshi Oka's Centennial Birthday, Kyoto/Nara (2001, 2004), Adv. Stud. in Pure Math., 42 (2004), pp. 203-207.

[O-1] T. Ohsawa, A reduction theorem for cohomology groups of very strongly q-convex Kähler manifolds, Invent. Math., 63 (1981), 335-354. Addendum: Invent. Math., 66 (1982), 391-393.

[O-2] T. Ohsawa, On the Levi-flats in complex tori of dimension two, Publ. RIMS. Kyoto Univ., 42 (2006), 361-377. Supplement: ibid. 379-382.

[O-3] T. Ohsawa, On the complement of Levi-flats in Kähler manifolds of dimension $\geq 3$, Nagoya Math. J., 185 (2007), 161-169.

[O-4] T. Ohsawa, A remark on pseudoconvex domains with analytic complements in compact Kähler manifolds, J. Math. Kyoto Univ., 47 (2007), 115-119.

[O-5] T. Ohsawa, $\bar{\partial}$-cohomology and geometry of the boundary of pseudoconvex domains, Ann. Polon. Math., 19 (2007), 249-262.

Added in proof. Examples in the appendix has already been known by E. Ghys, essentially (cf. Ann. Fac. Sci. Toulouse Math. (6), 5 (1996), 493-519). As for a somewhat more advanced result for (singular) foliations on tori, the reader is recommended to read M. Brunella: Codimension one foliations on complex tori, preprint. (See also Brunella's another paper in Indiana Univ. Math. J., 57 (2008), 31013114.)

Graduate School of Mathematics

Nagoya University

Furocho, Nagoya, 464-8602

Japan

ohsawa@math.nagoya-u.ac.jp 